\title{
Condiciones óptimas para almacenamiento del polen de Ochroma pyramidale
}

\author{
Optimal conditions for pollen storage of Ochroma pyramidale
}

\author{
Ivonne Jalca Zambrano a , Luz Cecilia García Cruzatty b,c*, Jessenia Castro Olaya ${ }^{\text {b, }}$ \\ Ronald Villamar Torres ${ }^{\text {, }}$, Marcelino Guachambala Cando ${ }^{\mathrm{e}}$

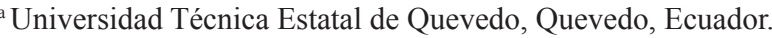

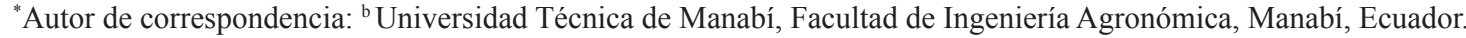 \\ ' Universidad Técnica Estatal de Quevedo, Facultad de Ciencias Ambientales, Quevedo, Ecuador, cecilialuz29@hotmail.com \\ ${ }^{\mathrm{d}}$ Université de Montpellier, CIRAD, INRA, Montpellier SupAgro, Montpellier, France. \\ ${ }^{\text {e }}$ Empresa $3^{\mathrm{a}}$ Composites, Departamento de Investigación y Desarrollo, Quevedo, Ecuador.
}

\begin{abstract}
SUMMARY
Knowledge about pollen biology is fundamental for the planning of controlled crosses in programs of conservation and genetic improvement of plant species. The objectives of this research were to determine the ideal medium for in vitro germination of pollen and to evaluate the effect of temperature and drying on the viability of stored pollen from Ochroma pyramidale. Pollen was obtained from flowers collected before anthesis. The appropriate culture medium for in vitro germination of pollen was determined by testing modifications of the medium proposed by Brewbaker and Kwack. Pollen drying was performed during different times (60, 120, 180 and 240 minutes) at $29{ }^{\circ} \mathrm{C}$ and $45 \%$ relative humidity. These samples were stored under temperatures of $5,-20$ and $-196{ }^{\circ} \mathrm{C}$, and evaluated for 45 days. The ideal medium for pollen germination was composed of $10 \%$ sucrose, $30 \mathrm{mg} \mathrm{L}^{-1}$ boric acid, $430 \mathrm{mg} \mathrm{L}^{-1}$ calcium nitrate. The initial viability calculated by in vitro germination was high, which indicates that the individuals evaluated can be used as pollen donors in the controlled crossing plans. Humidity below $5 \%$ allowed keeping the pollen stored, independently of the storage temperature during the 45 days of the test, without significant decrease in their viability and germination rate. However, pollen viability decreased rapidly when it was stored with high moisture content, regardless of storage temperatures.
\end{abstract}

Key words: in vitro germinability, pollen cryopreservation.

\section{RESUMEN}

El conocimiento sobre la biología del polen es fundamental para la planificación de cruzamientos controlados en programas de conservación y mejoramiento genético de especies vegetales. Esta investigación tuvo como objetivos determinar el medio idóneo para la germinación in vitro de polen y evaluar el efecto de temperatura y secado en la viabilidad de polen almacenado de Ochroma pyramidale. El polen se obtuvo de flores colectadas previo a la antesis. El medio de cultivo adecuado para realizar la germinación in vitro del polen se determinó probando modificaciones del medio propuesto por Brewbaker y de Kwak (BK). El secado del polen se realizó durante diferentes tiempos $\left(60,120,180\right.$ y 240 minutos) a $29{ }^{\circ} \mathrm{C}$ y $45 \%$ de humedad relativa; estas muestras fueron almacenadas bajo temperaturas de $5^{\circ},-20^{\circ} \mathrm{C}$ y $-196{ }^{\circ} \mathrm{C}$, y evaluadas durante 45 días. El medio idóneo para la germinación del polen estuvo compuesto por $10 \%$ de sacarosa, $30 \mathrm{mg} \mathrm{L}^{-1}$ ácido bórico, $430 \mathrm{mg} \mathrm{L}^{-1}$ nitrato de calcio. La viabilidad inicial calculada mediante germinación in vitro fue alta, lo que indica que los individuos evaluados se pueden utilizar como donantes de polen en los planes de cruzamiento controlado. La humedad por debajo del $5 \%$ permitió mantener el polen almacenado, sin disminución significativa de su viabilidad y tasa de germinación, durante los 45 días del ensayo, independientemente de la temperatura de almacenamiento. No obstante, la viabilidad del polen disminuyó rápidamente cuando fue almacenado con alto contenido de humedad, independientemente de las temperaturas de almacenamiento.

Palabras clave: germinabilidad in vitro, crioconservación de polen.

\section{INTRODUCCIÓN}

Ochroma pyramidale (Cav. ex Lam.) Urb. es una especie forestal pionera de gran importancia comercial en Ecuador, principalmente en la provincia Los Ríos donde se obtiene el $95 \%$ de la producción mundial. No obstante, el $98,84 \%$ de los productores usan semillas que son reco- lectadas de árboles en sus propias plantaciones, sin ningún criterio de selección o adquieren plántulas en viveros locales, cuyos propietarios no certifican la calidad de las semillas ni su procedencia (González Osorio et al. 2010). A pesar que se ha documentado que la ganancia genética obtenida al utilizar material superior es mayor al $15 \%$ para los caracteres crecimiento en altura y crecimiento en diá- 
metro (DAP), así como mayor a un $35 \%$ en volumen por unidad de área, pocos investigadores han abordado en los últimos años el tema de la efectividad de la selección de árboles (Balcorta y Vargas 2004).

En el Ecuador existe desde el año 2013 el programa de incentivos forestales por parte del gobierno nacional, en el cual participan pequeños, medianos y grandes agricultores así como empresas forestales (MAGAP 2014). Uno de los limitantes para participar de este programa es la incidencia de una enfermedad denominada comúnmente "muerte regresiva" que se observa en varias especies: Tectona grandis L., O. pyramidale, Gmelina arborea Roxb., Schizolobium parahyba (Vell.) S.F. Blake (Castro 2016), justamente las especies consideradas en este programa (MAGAP 2014).

Mediante el almacenamiento de polen es posible disminuir riesgos fitosanitarios ya que raramente se transfieren plagas y/o enfermedades a través del polen (a excepción de algunas enfermedades ocasionadas por virus), lo que permite la seguridad en el traslado e intercambio de germoplasma (FAO et al. 2007). En programas de mejoramiento genético se considera una de las principales alternativas para la conservación de alelos, facilitando el cruzamiento controlado entre individuos de interés (Dafni y Firmage 2000, Ganeshan et al. 2008). En el Ecuador no se han realizado estudios referentes a la biología y manejo del polen en especies forestales, lo que ha conllevado a que la reproducción de $O$. pyramidale y de las otras especies comerciales mencionadas se realice con semillas de polinización abierta recolectadas en plantaciones, puesto que no hay una oferta de semillas de huertos y/o rodales semilleros. Se sugiere que una posible depresión endogámica causada por esta práctica, sería la causa de los actuales problemas fitosanitarios.

Para evaluar la viabilidad del polen se utilizan técnicas in vivo e in vitro, siendo la primera más confiable, sin embargo, las pruebas de germinación in vitro son más utilizadas debido a que son más sencillas y rápidas para ejecutar. En el medio de cultivo, las sustancias nutritivas y sacarosa son importantes para la germinabilidad. La adición de agar al medio de cultivo, aunque en algunas especies resulta ser un aporte sustancial, se ha demostrado que no condiciona el desarrollo del tubo polínico (García et al. 2012).

Desde el siglo pasado, se han probado varios métodos para almacenar polen en diferentes especies (Yates et al. 1991, Connor y Towill 1993, Rajasekharan et al. 1994, García et al. 2015) demostrando que la temperatura y el contenido de humedad son factores importantes que influyen en su conservación. Por ejemplo, ha sido posible prolongar la viabilidad del polen disminuyendo su contenido de humedad y bajo condiciones de crioconservación en varias especies, por ejemplo: Carya illinoensis (Wangenh.) K. Koch (Yates et al. 1991) $\left(-196{ }^{\circ} \mathrm{C}\right)$, Nothofagus alpina (García et al. 2015), algunas especies del género Pinus (Tighe 2004), especies del género Protea (Van der Walt y Littlejohn 1996). El contenido de agua es un parámetro que, de acuerdo con la comprensión actual, afecta directamente la viabilidad del polen. La necesidad de reducir el contenido de agua antes de la exposición a bajas temperaturas parece ser un aspecto que requiere especial atención en estudios actuales y futuros, aunque en casos especiales el contenido de agua no puede reducirse a un nivel crítico (Shivanna y Johri 1985).

En programas de mejoramiento genético es primordial asegurarse de la viabilidad del polen de los genotipos a utilizarse. Además, en programas avanzados se debe conocer las condiciones adecuadas para manejo y almacenamiento de polen, así poder planificar cruzamientos manuales entre genotipos seleccionados. En el presente estudio se plantearon los siguientes objetivos: establecer el medio idóneo para evaluar la viabilidad del polen de $O$. pyramidale y determinar las condiciones de temperatura y contenido de humedad adecuados para su almacenamiento. La hipótesis planteada fue que es posible prolongar la viabilidad del polen de $O$. pyramidale en condiciones de crioconservación cuando se reduce su contenido de humedad a porcentajes muy bajos.

\section{MÉTODOS}

Fuente y extracción de polen. El material vegetal utilizado en los diferentes ensayos se obtuvo en un huerto semillero, constituido por progenies de 30 árboles de 4,2 años de edad, ubicado en la hacienda "Madera Seca 14" (MS14), propiedad de la empresa 3A Composites, ubicada en el cantón El Empalme, sector Los Bancos, provincia del Guayas, Ecuador, entre las coordenadas $1^{\circ} 2^{\prime} 57,43^{\prime \prime}$ latitud Sur y $79^{\circ} 35^{\prime} 33,22^{\prime \prime}$ longitud Oeste. Según la clasificación de Holdrige, esta área corresponde a la clasificación ecológica bosque seco tropical.

Se recolectaron un total de 50 flores (cinco flores por 10 árboles) en horario de 15:00 a 17:00 h, justo antes de su apertura, considerando que $O$. pyramidale, cuya floración ocurre durante todo el año, presenta antesis nocturna que inicia a las 18:00 h. Estas flores se mezclaron y se dividieron en tres grupos: un primer grupo de 10 flores cuyo polen fue utilizado para determinar el medio adecuado para evaluar la viabilidad del polen, un segundo grupo de 10 flores que se utilizó para la determinación del contenido total de humedad del polen (extraído inmediatamente después de la colecta de las flores) y un tercer grupo de 30 flores que se utilizó para el establecimiento de los ensayos de secado y almacenamiento. Para evitar la deshidratación durante el transporte, las flores se guardaron en bolsas de papel kraft y se colocaron en hieleras $\left( \pm 20^{\circ} \mathrm{C}\right)$.

Los ensayos de almacenamiento y respectivas evaluaciones de germinabilidad se realizaron en el Laboratorio de Genética de la empresa 3A Composites. Para la extracción del polen las flores se mantuvieron a temperatura ambiente $\left(28-30^{\circ} \mathrm{C}\right)$ hasta que ocurrió la dehiscencia de las anteras y la consecuente liberación del polen. El polen se separó de las anteras con una espátula, y se colocó en platos Petri. No hubo necesidad de realizar filtrado ni lim- 
pieza. Finalmente se mezcló el polen obtenido de las flores para formar una muestra homogénea.

Formulación y evaluación de los medios de germinación. Para determinar el medio adecuado para evaluar la viabilidad de polen de $O$. pyramidale, mediante germinación in vitro, se probaron seis medios de cultivo líquidos: el medio establecido por Brewbaker y Kwack (1963) (M1) y cinco medios que corresponden a modificaciones del Medio M1 (cuadro 1).

Inmediatamente después de que el polen fue extraído se realizó la siembra. Se colocaron $10 \mathrm{ml}$ de los medios mencionados en cajas Petri $(\varnothing 5 \mathrm{~cm})$. El conteo de granos de polen se realizó bajo microscopio óptico con un aumento de 40x. La germinación de los granos de polen comenzó de 30 a 40 minutos después de la siembra, razón por la que se fijó las muestras a los 60 minutos aplicando azul de anilina en lactofenol, pues el rápido desarrollo de los tubos polínicos impedía el conteo de los granos de polen y la respectiva evaluación.

Se colocaron cuatro repeticiones por medio de cultivo (tratamientos) y se realizaron cuatro observaciones por cada repetición, considerando que en el área observada hubiera al menos 50 granos de polen. Al no encontrarse diferencia entre los medios evaluados, para discriminar entre estos se realizó el mismo proceso de evaluación de medios después de 24 horas de la colecta del polen. Los datos generados se arreglaron bajo un diseño completamente al azar. Se realizó análisis de varianza, previo al análisis de homecedasticidad utilizando el test de Bartlett. Posteriormente se realizó la comparación de medias a través de la prueba de Tukey al $5 \%$ de probabilidad.

Cuadro 1. Medios de cultivo probados para evaluar la germinabilidad del polen de Ochroma pyramidale.

Culture media tested to evaluate the germination of pollen of Ochroma pyramidale.

\begin{tabular}{cccccc}
\hline & $\begin{array}{c}\text { Sacarosa } \\
(\%)\end{array}$ & $\begin{array}{c}\mathrm{H}_{3} \mathrm{~B}_{3} \\
\left(\mathrm{mg} \mathrm{L}^{-1}\right)\end{array}$ & $\begin{array}{c}\mathrm{Ca}\left(\mathrm{NO}_{3}\right)_{2} \\
\left(\mathrm{mg} \mathrm{L}^{-1}\right)\end{array}$ & $\begin{array}{c}\mathrm{MgSO}_{4} \\
\left(\mathrm{mg} \mathrm{L}^{-1}\right)\end{array}$ & $\begin{array}{c}\mathrm{KNO}_{3} \\
\left(\mathrm{mg} \mathrm{L}^{-1}\right)\end{array}$ \\
\hline M1 & 10 & 100 & 300 & 200 & 100 \\
M2 & 5 & 50 & 150 & 100 & 50 \\
M3 & 10 & 30 & 430 & - & - \\
M4 & 5 & 20 & 200 & - & - \\
M5 & 10 & 30 & - & - & - \\
M6 & 5 & 20 & - & - & - \\
\hline
\end{tabular}

Contenido de humedad y almacenamiento del polen. Para la extracción y manejo del polen se siguió con la metodología de García et al. (2015), aunque se probaron diferentes tiempos y temperatura de secado. Para calcular el contenido total de agua en polen fresco de $O$. pyramidale, el total de polen extraído se homogenizó, se dividió en tres partes iguales (muestras de $490 \mathrm{mg}$ ) y se colocó en estufa a $60{ }^{\circ} \mathrm{C}$, en placas Petri durante una hora. Tanto para calcular el contenido de agua del polen fresco, como para calcular la pérdida de agua después de la deshidratación, el polen fue pesado utilizando una balanza analítica, antes y después del secado. El contenido de agua de una muestra se calculó a partir de la pérdida de peso, teniendo como base su peso fresco (García et al. 2015).

Para el ensayo de almacenamiento, se mezcló el polen extraído para obtener una muestra homogénea. Se probaron diferentes tiempos de secado: 60, 120, 180 y $240 \mathrm{mi}-$ nutos. El secado se realizó colocando las placas Petri con $0,88 \mathrm{~g}$ de polen dentro de una estufa Memmert con humedad relativa de $45 \%$ y temperatura de $29{ }^{\circ} \mathrm{C}$. Se calculó la viabilidad de todas las muestras de polen mediante germinación in vitro, antes y después del secado, para determinar los efectos del contenido de humedad en la longevidad del polen almacenado.

Después del secado se colocaron muestras de polen (20 mg) en tubos Eppendorf ( $0,25 \mathrm{~mL})$, y estos a su vez se guardaron en tubos Falcon $(15 \mathrm{~mL})$. Para las muestras de polen almacenado a $5{ }^{\circ} \mathrm{C}$ y $-20{ }^{\circ} \mathrm{C}$, se colocaron $800 \mathrm{mg}$ de dióxido de silicio (comercialmente sílica gel) en el fondo de los tubos Falcon y algodón para evitar el contacto directo con las muestras. En el caso de las muestras almacenadas a $-196{ }^{\circ} \mathrm{C}$ los tubos Eppendorf se envolvieron en papel aluminio e igualmente se los introdujo en tubos Falcon pero sin sílica gel.

Las muestras de polen almacenadas, se evaluaron a los 15,30 y 45 días, previo a procesos de aclimatación y rehidratación mediante el aumento de temperatura en forma gradual, de la siguiente forma: las muestras almacenadas a $5{ }^{\circ} \mathrm{C}$ se dejaron en reposo por lapso dos horas a $13{ }^{\circ} \mathrm{C}$ y después una hora a $25^{\circ} \mathrm{C}$ y $100 \%$ de humedad relativa; las muestras almacenadas a $-20{ }^{\circ} \mathrm{C} \mathrm{y}-196{ }^{\circ} \mathrm{C}$ se colocaron dos horas a $0{ }^{\circ} \mathrm{C}$ y luego se aumentó la temperatura gradualmente hasta llegar a la rehidratación a $25^{\circ} \mathrm{C}$ y $100 \%$ de humedad relativa.

Se analizó el efecto del contenido de humedad y la temperatura de almacenamiento sobre la longevidad de polen almacenado con diferentes contenidos de humedad, utilizando la prueba de Tukey $5 \%$ de probabilidad, posterior al respectivo análisis de varianza.

\section{RESULTADOS}

El mayor porcentaje de germinabilidad del polen de O. pyramidale se obtuvo utilizando el M4; sin embargo, no hubo diferencias con otros cinco medios utilizados, a excepción del M5 con el cual se obtuvo el más bajo porcentaje de germinación (cuadro 2). No obstante, se escogió el M3 para estudiar el polen de esta especie pues se logró el mayor porcentaje de germinación (50 \%) a 24 horas después de la colecta. 
El contenido total de humedad $(\mathrm{CH})$ del polen de $O$. pyramidale fue de $49,8 \%( \pm 0,43)$, el mismo que se redujo drásticamente cuando el polen fue sometido a diferentes tiempos de secado. Se perdió aproximadamente el $45 \%$ del contenido de humedad al pasar por 180 y 240 minutos de secado, lo que significó una pérdida del contenido de humedad inicial por encima del $90 \%$ quedando con el 4,9 y $4,5 \%$, respectivamente (cuadro 3 ).

El contenido de humedad en el polen se redujo a medida que aumentó el tiempo de secado, de un total del $50 \%$ a menos del $5 \%$ después de los 240 minutos de secado. Sin embargo, el proceso de secado no resultó deletéreo para el polen, pues la germinación, que en polen fresco fue de un $65,62 \%$ no disminuyó significativamente, después de 60 y 240 minutos de secado respectivamente (figura 1).

Los granos de polen secados durante 60 minutos, almacenados a $5{ }^{\circ} \mathrm{C},-20{ }^{\circ} \mathrm{C}$ y $-196{ }^{\circ} \mathrm{C}$, perdieron su viabilidad a los 45 días. Las muestras secadas por 120 minutos perdieron la viabilidad almacenadas a $5{ }^{\circ} \mathrm{C}$, mientras que a temperaturas de -20 y $-196{ }^{\circ} \mathrm{C}$ presentaron menos del $6 \%$ de granos viables. Se logró prolongar exitosamente la longevidad del polen de $O$. pyramidale, almacenándolo con < $5 \%$ de contenido de humedad, independientemente de la temperatura, pues bajo estas condiciones más del $90 \%$ de los granos de polen pudieron germinar durante los tiempos evaluados (cuadro 4, figura 2).

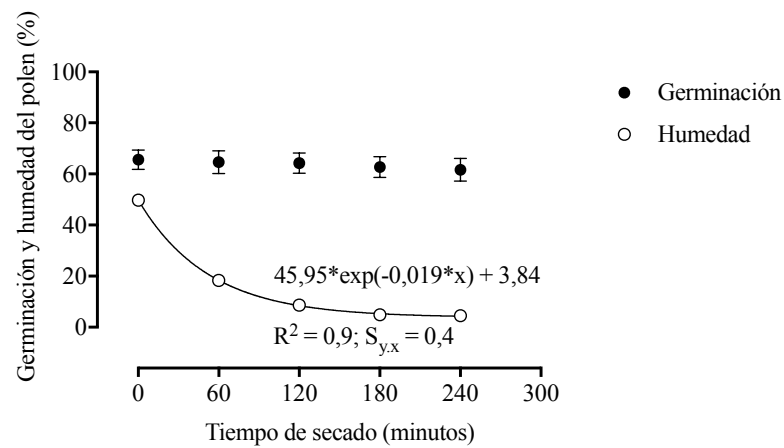

Figura 1.Efecto del tiempo de secado sobre el contenido de humedad y germinación del polen de Ochroma pyramidale. Las barras indican el error estándar.

Effect of drying time on moisture content and viability of Ochroma pyramidale pollen.

Cuadro 2. Germinabilidad de polen de Ochroma pyramidale en diferentes medios de cultivo.

Pollen germinability of Ochroma pyramidale in different culture media.

\begin{tabular}{ccc}
\hline Tratamientos & \% germinación inicial & \% germinación de polen almacenado 24 horas \\
\hline M1 & $69,55 \mathrm{a}$ & $33,16 \mathrm{~b}$ \\
M2 & $56,00 \mathrm{a}$ & $0,00 \mathrm{~d}$ \\
M3 & $70,14 \mathrm{a}$ & $50,39 \mathrm{a}$ \\
M4 & $72,72 \mathrm{a}$ & $16,20 \mathrm{c}$ \\
M5 & $22,17 \mathrm{~b}$ & $0,00 \mathrm{~d}$ \\
M6 & $56,80 \mathrm{a}$ & $0,00 \mathrm{~d}$ \\
\hline
\end{tabular}

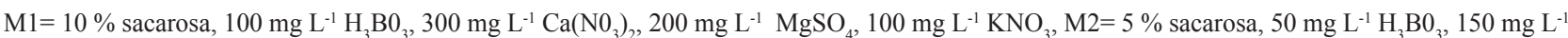

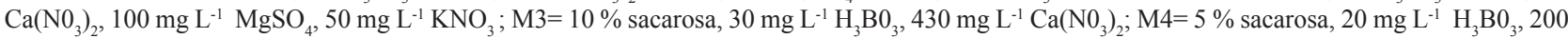
$\mathrm{mg} \mathrm{L}^{-1} \mathrm{Ca}\left(\mathrm{NO}_{3}\right)_{2}$, M5 $=10 \%$ sacarosa, $30 \mathrm{mg} \mathrm{L}^{-1} \mathrm{H}_{3} \mathrm{~B}_{3}$, $\mathrm{T} 6=5 \%$ sacarosa, $20 \mathrm{mg} \mathrm{L}^{-1} \mathrm{H}_{3} \mathrm{~B}_{3}$

"Medias con letras iguales no presentan diferencia estadística (Tukey, $P<0,05$ ).

Cuadro 3. Contenido de humedad $(\mathrm{CH})$ del polen de Ochroma pyramidale después de diferentes tiempos de secado.

Moisture content of Ochroma pyramidale pollen after different drying times.

\begin{tabular}{|c|c|c|c|c|c|}
\hline $\begin{array}{l}\text { Tiempo de secado } \\
\text { (minutos) }\end{array}$ & $\begin{array}{l}\text { Peso inicial } \\
(\mathrm{mg})\end{array}$ & $\begin{array}{l}\text { Peso final } \\
\quad(\mathrm{mg})\end{array}$ & $\begin{array}{c}\% \mathrm{CH} \text { perdido respecto } \\
\text { del peso total }\end{array}$ & $\% \mathrm{CH}$ restante & $\begin{array}{l}\% \mathrm{CH} \text { perdido respecto del } \\
\text { contenido de agua original }\end{array}$ \\
\hline 60 & 490,00 & 335,58 & 31,51 & 18,28 & 63,28 \\
\hline 120 & 490,00 & 288,78 & 41,07 & 8,73 & 82,46 \\
\hline 180 & 490,00 & 335,58 & 44,90 & 4,89 & 90,16 \\
\hline 240 & 490,00 & 288,78 & 45,34 & 4,46 & 91,04 \\
\hline
\end{tabular}

"Contenido de humedad inicial 49,8\%. 
Cuadro 4. Viabilidad del polen de Ochroma pyramidale almacenado bajo diferentes condiciones de humedad $(\mathrm{CH})$ y temperatura. Viability of Ochroma pyramidale pollen stored under different conditions of moisture content and temperature.

\begin{tabular}{|c|c|c|c|c|c|c|}
\hline \multirow{2}{*}{$\begin{array}{l}\text { Tiempo de secado } \\
\text { (minutos) }\end{array}$} & \multirow{2}{*}{$\begin{array}{c}\text { Germinación } \\
\qquad \%)\end{array}$} & \multirow[t]{2}{*}{$\mathrm{CH}(\%)$} & \multirow{2}{*}{$\begin{array}{c}\text { Temperatura } \\
\left({ }^{\circ} \mathrm{C}\right)\end{array}$} & \multicolumn{3}{|c|}{ \% de germinación } \\
\hline & & & & 15 días & 30 días & 45 días \\
\hline \multirow{3}{*}{0} & \multirow{3}{*}{$65,62( \pm 3,76)$} & \multirow{3}{*}{49,8} & 5 & $0 \mathrm{~d}$ & $0 \mathrm{f}$ & $0 \mathrm{c}$ \\
\hline & & & -20 & $0 \mathrm{~d}$ & $0 \mathrm{f}$ & $0 \mathrm{c}$ \\
\hline & & & -196 & $0 \mathrm{~d}$ & $0 \mathrm{f}$ & $0 \mathrm{c}$ \\
\hline \multirow{3}{*}{60} & \multirow{3}{*}{$64,64( \pm 4,49)$} & \multirow{3}{*}{18,28} & 5 & $41,71( \pm 4,05) \mathrm{c}$ & $6,73( \pm 2,57) \mathrm{e}$ & $0 \mathrm{~b}$ \\
\hline & & & -20 & $46,27( \pm 2,68) b c$ & $17,65( \pm 3,28) d$ & $0 \mathrm{~b}$ \\
\hline & & & -196 & $44,63( \pm 4,67) \mathrm{bc}$ & $44,63( \pm 3,88) b c$ & $0 \mathrm{~b}$ \\
\hline \multirow{3}{*}{120} & \multirow{3}{*}{$64,23( \pm 3,98)$} & \multirow{3}{*}{8,73} & 5 & $47,10( \pm 2,47) b c$ & $25,65( \pm 10,37) d$ & $0 \mathrm{~b}$ \\
\hline & & & -20 & $44,07( \pm 5,70) \mathrm{bc}$ & $15,90( \pm 3,07) \mathrm{de}$ & $5,61( \pm 3,28) b$ \\
\hline & & & -196 & $45,33( \pm 7,48) b c$ & $39,63( \pm 5,53) \mathrm{c}$ & $4,65( \pm 3,59) b$ \\
\hline \multirow{3}{*}{180} & \multirow{3}{*}{$62,76( \pm 4,08)$} & \multirow{3}{*}{4,89} & 5 & $61,75( \pm 2,37) \mathrm{a}$ & $53,15(2,69) \mathrm{a}$ & $50,25( \pm 5,77) \mathrm{a}$ \\
\hline & & & -20 & $52,57( \pm 2,70)$ a & $57,03( \pm 5,19)$ a & $51,10( \pm 4,01) \mathrm{a}$ \\
\hline & & & -196 & $64,26( \pm 3,07)$ a & $63,96( \pm 2,91)$ a & $52,88( \pm 6,51) \mathrm{a}$ \\
\hline \multirow{3}{*}{240} & \multirow{3}{*}{$61,65( \pm 4,48)$} & \multirow{3}{*}{4,46} & 5 & $61,66( \pm 1,57)$ a & $59,54( \pm 0,93)$ a & $58,9( \pm 6,74) \mathrm{a}$ \\
\hline & & & -20 & $65,27( \pm 2,22)$ a & $63,58( \pm 4,56) \mathrm{a}$ & $58,24( \pm 6,47) \mathrm{a}$ \\
\hline & & & -196 & $64,68( \pm 1,30)$ a & $63,44( \pm 1,88)$ a & $57,9( \pm 6,23) \mathrm{a}$ \\
\hline
\end{tabular}

Medias con letras en común no son significativamente diferentes (Tukey, $P<0,05$ ).

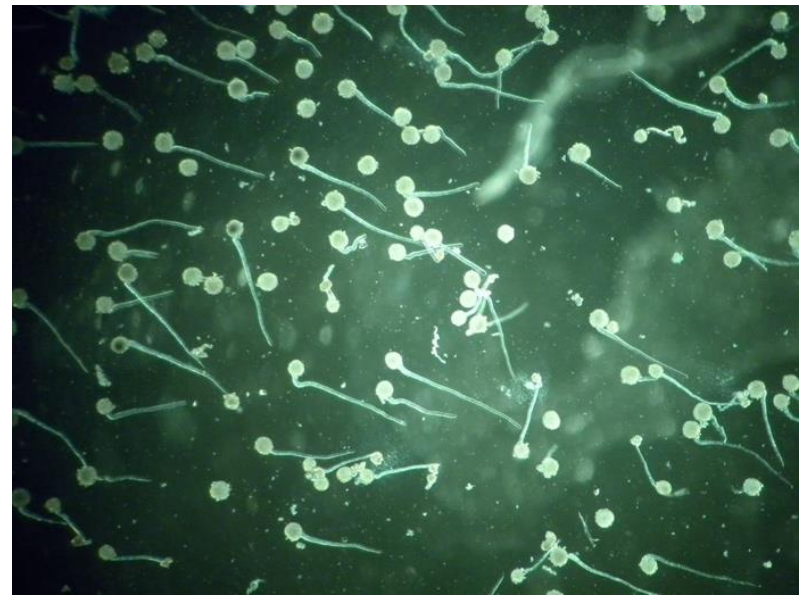

Figura 2. Germinación in vitro de polen conservado por 45 días a $-196{ }^{\circ} \mathrm{C}$.

In vitro germination of conserved pollen for 45 days at $-196^{\circ} \mathrm{C}$.

\section{DISCUSIÓN}

El presente trabajo constituye una primera aproximación para el manejo de polen $O$. pyramidale, pues no existen trabajos publicados relacionados a germinación, viabi- lidad, almacenamiento o alguna otra temática referente a la biología del polen de esta especie. La germinación inicial del polen de $O$. pyramidale fue alta $(>70 \%$ ). La viabilidad del polen se considera una medida de la fertilidad masculina; por lo tanto, el polen de los genotipos evaluados puede utilizarse en los programas de mejoramiento genético, para realizar cruzamientos controlados entre genotipos de interés económico.

Para evaluar la viabilidad del polen de esta especie se utilizó un medio líquido constituido por $10 \%$ sacarosa y micronutrientes $\left(\mathrm{H}_{3} \mathrm{BO}_{3}, \mathrm{Ca}\left(\mathrm{NO}_{3}\right)\right)$ con el cual se obtuvieron mejores resultados en relación a los otros medios evaluados; el polen de esta especie no germina en medios sólidos según pre ensayos realizados. Como los granos de polen son liberados bajo condiciones de desecación, la tasa de hidratación es crítica para la germinación del polen, particularmente para polen disecado. En un medio líquido, la hidratación ocurre en pocos minutos. El boro, por su efecto estimulatorio en la germinación de granos de polen, es un componente regular de los medios de germinación. Se cree que el polen es deficiente en boro lo cual se compensa con altos niveles de este elemento en el estigma. Se sugiere que el calcio es primordial para la germinación del polen de $O$. pyramidale, pues entre los medios evaluados la germinación fue baja en el único que no contenía calcio. En algunas especies el calcio no es requerido para la 
germinación del polen porque los granos de polen ya contienen altos niveles de calcio endógeno (Shivanna 2003).

Hilo et al. (2015) determinaron que los azúcares en el medio de cultivo cumplen un rol primordial en la regulación de la concentración osmótica durante la germinación de los granos de polen y sirven como material nutriente para el crecimiento de los tubos polínicos. Resultados similares a los de este estudio se obtuvieron en la especie relacionada Theobroma cacao L. (Malvaceae) (Esteves et al. 2009), índices de germinación in vitro de granos de polen por encima del $70 \%$, utilizando un medio con los mismos componentes pero en diferentes proporciones. También en otras angiospermas como Aechmea bicolor L.B. Smith (De Souza et al. 2015) se ha logrado la germinación in vitro del polen utilizando medios similares. Según Shivanna (2003), el tipo y concentración requerida de azúcar para la germinación del polen es menos específica en la presencia de boro, lo que explicaría la alta germinación de los granos de polen en los medios utilizados independientemente de las diferentes concentraciones de agar y sacarosa.

Se logró prolongar la viabilidad del polen de $O$. pyramidale reduciendo el contenido de humedad por debajo del $5 \%$, independientemente de las temperaturas de almacenamiento; bajo estas condiciones, prácticamente, no existe agua libre para el transporte intracelular (Shivanna y Jhori 1985, García et al. 2015). Por otra parte, un elevado contenido de humedad permite que continúe la actividad metabólica y se pierda la viabilidad a medida que transcurre el tiempo de almacenamiento. Por el contrario, cuando los granos de polen con alto contenido de humedad son almacenados a temperaturas ultrabajas ocurre la formación de cristales de hielo y por ende el rompimiento de las membranas (Shivanna y Jhori 1985, García et al. 2015), lo que explicaría la pérdida total de la viabilidad en todas las muestras almacenadas con mayor contenido de humedad.

Las muestras de polen de $O$. pyramidale almacenadas con menor contenido de humedad se mantuvieron viables a diferentes condiciones de temperatura, lo cual se puede atribuir a la disminución de la tasa de respiración y por ende al menor gasto energético (Stanley y Linskens 1974). Los resultados indican que las condiciones de secado permitieron mantener la estabilidad de las membranas en los granos de polen deshidratados, es decir, no hubo una alteración irreversible en su estructura, por lo pudieron regenerarse con la rehidratación y recuperar sus funciones normales (Shivanna y Heslop-Harrison 1981, Shivanna y Sawhney 2005, García et al. 2015).

\section{CONCLUSIONES}

El contenido total de humedad del polen de $O$. pyramidale es similar a otras especies vegetales. Su viabilidad se puede evaluar a través de germinabilidad in vitro utilizando un medio líquido. La viabilidad inicial del polen calculada mediante este método fue alta, lo que indica que los individuos evaluados se pueden utilizar como donantes de polen en los planes de cruzamiento controlado. Es posible prolongar la viabilidad del polen de $O$. pyramidale cuando es sometido a un alto grado de deshidratación, independientemente de las temperaturas de almacenamiento.

\section{REFERENCIAS}

Balcorta Martínez HC, JJ Vargas Hernández. 2004. Variación fenotípica y selección de árboles en una plantación de melina (Gmelina arborea Linn., Roxb.) de tres años de edad. Revista Chapingo. Serie Ciencias Forestales y del Ambiente 10 (1): 13-19.

Brewbaker J, B Kwack. 1963. The essential role of calcium ion in pollen germination and pollen tube growth. American Journal of Botany 50(9): 859-65. DOI: 10.2307/2439772

Castro J. 2016. Aspectos biológicos y ecológicos de Coptoborus ochromactonus Smith y Cognato (Coleoptera: Scolytinae), y la relación de sus hongos asociados en la muerte regresiva de Ochroma pyramidale (Cav. Ex. Lam.) Urb. Tesis Doctoral. Valdivia, Chile. Facultad de Ciencias Forestales y Recursos Naturales, Universidad Austral de Chile. 149 p. http://cybertesis.uach.cl/tesis/uach/2016/egc355a/doc/ egc355a.pdf

Connor K, L Towill. 1993. Pollen handling protocol and hydration/dehydration characteristics of pollen application to long term storage. Euphytica 68: 77-84. DOI: $10.1007 /$ BF00024157

Dafni A, D Firmage. 2000. Pollen viability and longevity: practical, ecological and evolutionary implications. Plant Systematics and Evolution 222: 113-4. DOI: 10.1007/ BF00984098

De Souza E, F Souza, M Rossi, N Brancalleão, C da Silva, A Martinelli. 2015. Viability, storage and ultrastructure analysis of Aechmea bicolor (Bromeliaceae) pollen grains, an endemic species to the Atlantic forest. Euphytica 204(1): 13-28. DOI: 10.1007/s10681-014-1273-3

Esteves P, M Souza, F Roza, P Lawinscky, I Santos, D Ahnert. 2009. Performance polínica em cacaueiros (Theobroma cacao L.) autocompatíveis e autoincompatíveis. Brazilian Journal of Botany 32(3): 617-20. DOI: 10.1590/S0100$\underline{84042009000300019}$

FAO (Organización de las Naciones Unidas para la Agricultura y la Alimentación, IT), FLD (Bosque y Paisaje de Dinamarca), Bioversity International. 2007. Conservación y manejo de recursos genéticos forestales. Vol. 3: En plantaciones y bancos de germoplasma (ex situ). Roma, Italia. Instituto Internacional de Recursos Fitogenéticos. 98 p. Disponible en: https://www.bioversityinternational.org/fileadmin/_migrated/uploads/tx_news/Conservaci $\% \mathrm{C} 3 \% \mathrm{~B} 3 \mathrm{n}$ y_manejo de los recursos gen $\%$ C $3 \%$ A 9 ticos forestales 1297.pdf

García J, C Rejón, J Ramírez, A Alché, M Rodriguéz. 2012. Evaluación de diferentes métodos para estimar la calidad del polen en distintos cultivares de olivo (Olea Europaea L.). Polen 20(0): 60-72. DOI: 10.14201/pol.v20i0.8921

García Cruzatty L, M Rivero, F Droppelmann. 2015. Effect of temperature and drying on the longevity of stored Nothofagus alpina pollen. New Zealand Journal of Botany 53(3): 155-64. DOI: 10.1080/0028825X.2015.1045528

Ganeshan S, P Rajasekharan, S Shashikumar, W Decruze. 2008. 
Cryopreservation of pollen. In Reed BM ed. Plant cryopreservation: a practical guide. New York, USA. Springer. $p$. 443-447. DOI: $10.1007 / 978-0-387-72276-4 \quad 17$

González Osorio B, X Cervantes, E Torres, C Sánchez, L Simba. 2010. Caracterización del cultivo de balsa (Ochroma pyramidale) en la Provincia de los Ríos, Ecuador. Ciencia y Tecnología 3(2): 7-11. DOI: $10.18779 /$ cyt.v3i2.45

Hilo E, F Duarte, M Lanzoni, N Brancalleão, C A da Silva Ledo, A Pinheiro. 2015. Viability, storage and ultrastructure analysis of Aechmea bicolor (Bromeliaceae) pollen grains, an endemic species to the Atlantic forest. Euphytica 204(1): 13-28. DOI: $10.1007 / \mathrm{s} 10681-014-1273-3$

Rajasekharan P, T Rao, T Janakiram, S Ganeshan. 1994. Freeze preservation of Gladiolus pollen. Euphytica 80: 105-109. DOI: $10.1007 / \mathrm{BF} 00039304$

MAGAP (Ministerio de Agricultura, Ganadería, Acuacultura y Pesca, EC). 2014. Programa de Incentivos para la Reforestación con Fines Comerciales. Guayaquil, Ecuador. MAGAP. 38 p.

Shivanna K, J Heslop-Harrison. 1981. Membrane state and pollen viability. Annals of Botany 47(6): 759-770. DOI: 10.1093/oxfordjournals.aob.a086075

Shivanna K, B Jhori. 1985. The Angiosperm pollen: structure and function. New Delhi, India. Wiley. 374 p.

Shivanna K. R. 2003. Pollen biology and biotechnology. Science Publishers, Universidad de Michigan. 301 p.
Shivanna K, V Sawhney. 2005. Pollen biotechnology for crop production and improvement. Cambridge, England. Cambridge University Press. $531 \mathrm{p}$.

Stanley R, H Linskens. 1974. Pollen: Biology, Biochemistry and Management. New York, USA. Springer Verlag. DOI: $\underline{10.21273 / \mathrm{JASHS} .116 .3 .430}$

Suárez C. 2009. Caracterizacion Estructural e Histoquimica del Pistilo durante la fase progamica e implicacion de pectinas y AGPs en las interacciones polen-pistilo en el olivo. Tesis Doctoral Universidad de Granada. Departamento de Fisiología Vegetal. 247 p. Disponible en: http://digibug.ugr.es/ handle/10481/2388.

Tighe M. 2004. Manual de recolección y manejo de polen de pinos tropicales y subtropicales procedentes de rodales naturales. Raleigh, NC, USA. CAMCORE. 22 p. Disponible en: https://www.academia.edu/4075259/Manual de Recolecci\%C3\%B3n y Manejo de Polen de Pinos Tropicales_y_Subtropicales_Procedentes de Rodales_Naturales CAMCORE

Van der Walt I, G Littlejohn. 1996. Storage and viability testing of Protea pollen. Journal of the American Society for Horticultural Science 121(5): 804-9.

Yates I, D Sparks, K Connor, L Towill. 1991. Reducing pollen moisture simplifies long term storage of pecan pollen. American Society for Horticultural Science 116: 430-434. DOI: $\underline{10.21273 / J A S H S .116 .3 .430}$ 
